\title{
Anterior Cervical Discectomy and Fusion with Stand-Alone Trabecular Metal Cages as a Surgical Treatment for Cervical Radiculopathy: Mid-Term Outcomes
}

\author{
Khaldoun ElAbed, Ahmad Shawky, Mo Barakat, Donald Ainscow \\ Department of Orthopaedics, Alzahra Hospital, Sharjah, UAE
}

Study Design: Retrospective case cohort study done between 2002 and 2012.

Purpose: To assess the mid-term clinical and radiological outcomes of 1-level and 2-level anterior cervical discectomy and fusion (ACDF) with stand-alone trabecular metal cages.

Overview of Literature: ACDF is the gold standard surgical treatment for cervical degenerative disease. The usual surgical practice is to use an anteriorly placed fusion plate with or without interdiscal cages.

Methods: Patients between 36 and 64 years of age diagnosed with cervical radiculopathy who underwent ACDF using stand-alone trabecular metal cages with at least 3 years follow-up were included in this study. Recorded clinical outcomes included residual axial neck pain, radicular arm pain, upper extremity weakness, and upper extremity altered sensation. Visual Analogue scores were also recorded. Fusion was assessed by lateral radiographs looking for bone breaching and radiolucent lines around the device at the latest follow-up.

Results: Ninety patients were included in the study. Fifty-one patients underwent 2-level surgery and 39 patients underwent 1-level surgery. Mean age was $44 \pm 10.4$ years and mean follow-up time was $4.5 \pm 2.6$ years. Patients reported excellent or good outcomes $(90 \%)$, as well as improvements in axial neck pain (80\%), radicular arm pain (95\%), upper extremity weakness (85\%), and upper extremity altered sensation (90\%). Most patients (90\%) progressed to fusion at the 1-year follow-up. The reoperation rate was 3.6\%. There was no reported persistent dysphagia, voice complaints, dural tear, or tracheal or oesophageal perforation in any of the patients. One patient developed a deep methicillin-resistant Staphylococcus aureus infectious infarction of the spinal cord, which was treated with antibiotics. Recovery was complete at the 1-year follow up.

Conclusions: Mid-term results show that surgical treatment with ACDF with trabecular metal cages is a safe and effective treatment of single and 2-level cervical disc radiculopathy and neck pain.

Keywords: Anterior; Fusion; Cages

Received Jul 19, 2015; Revised Sep 26, 2015; Accepted Oct 01, 2015

Corresponding author: Mo Barakat

Department of Orthopaedics, Alzahra Hospital, Sharjah, UAE

E-mail: mo.barakat1976@gmail.com 


\section{Introduction}

Cervical radiculopathy is one of the most frequently occurring and treated cervical spinal conditions. Radiculopathy is a disorder of the cervical nerve root, manifesting usually as a combination of neck and upper limb pain, sensory deficit or motor disturbance [1].

Anterior cervical discectomy and fusion (ACDF) is the gold standard for the surgical treatment of cervical disease, with multiple ways of performing the procedure reported in the literature since its original description by Smith and Robinson [2-7]. ACDF using stand-alone cages packed with local autograft has been used with favorable results $[8,9]$. Over the last 10 years, the practice at our institute is to use stand-alone cages of trabecular metal (TM-S, Zimmer, Minneapolis, MN, USA) without an autograft. To our knowledge, there are no medium- to longterm outcome results of cervical trabecular metal cages without autograft reported in the literature.

The purpose of this study was to evaluate the clinical and radiological outcomes of our 10-year experience of ACDF using stand-alone trabecular metal cages in the treatment of cervical radiculopathy

\section{Materials and Methods}

Between July 2002 to July 2012, 90 consecutive patients between the ages of 36 and 64 years at surgery (mean, 44 years) who had been diagnosed with cervical radiculopathy and/or axial neck pain with sclerodermal distribution who underwent single or 2-level ACDF with trabecular metal cages and followed-up for a minimum of 3 years were included. Patients with a history of spine infection or tumor, cervical spine fractures, traumatic spinal cord injury, cervical myelopathy, or prior cervical spine surgery were excluded.

Two fellowship-trained spine surgeons performed the operations, with operation levels limited to the lower part of the cervical spine (C4-7). Two independent senior spine surgeons reviewed the patients and outcomes. We identified and included 90 consecutive patients who met all the inclusion and exclusion criteria with at least 3 years follow-up.

Clinical assessments of axial neck pain, radicular arm pain, upper extremity weakness, and upper extremity altered sensation were performed. As well visual analog scale (VAS) scores were determined preoperatively and postoperatively at 3 months, 1 year, 3 years, and at the latest postoperative follow-up. Radiological assessments were carried out at the same time periods. Axial neck pain and radicular arm pain were subjectively rated as improved or unchanged, upper extremity weakness and motor strength were objectively rated as normal or abnormal. Likewise, upper extremity sensory function was graded as altered to light touch, or normal. An incidence of new deficits was recorded for both motor and sensory function based on newly identified neurologic events.

\section{Statistical analysis}

The average, mean and standard deviation were calculated for the 90-patient series. Fisher exact test was utilized to compare the rates of categorical variables. The statistical significance was set at $p<0.05$.

\section{Results}

The mean patient age was 44 years (range, 37-62 years) with a mean follow-up of 4.5 years (range, 2-7 years). Fifty one patients underwent 2-level ACDF and 39 patients underwent 1-level ACDF. Mean operating time was 43 minutes per level (range, 35-50 minutes), and average blood loss was $120 \mathrm{~mL}$ per procedure (range, $83-180 \mathrm{~mL}$ ).

There were improvements in axial neck pain of $80 \%$ at the 1-year follow-up and 75\% at the 3-year follow-up, and in radicular arm pain with $95 \%$ at the 1-year followup, and $85 \%$ at the 3 -year follow-up. VAS scores improved from a preoperative mean of 3 to a mean of 9 at 1 year and a mean of 8 at 3 years postoperatively.

Preoperatively, $70 \%$ of patients had sensory deficit and $50 \%$ of patients had motor deficit. There was an improvement in preoperative sensory deficit at the 1-year followup (90\%) and at final follow-up (90\%). There was also an improvement in the preoperative motor weakness at the 1-year follow-up (95\%) and 3-year follow-up (85\%). Fifteen percent of the patients had a motor deficit at the latest follow-up, had undergone a 2-level ACDF, and had radiographic evidence of pseudoarthrosis (Fig. 1). This incidence was lower than the reported rates for anterior plating [10]. No cage extrusion or migration occurred. Subsidence occurred in $8 \%$ of levels fused with no correlation found with clinical outcomes.

Fusion was assessed using lateral radiographs and magnetic resonance imaging (MRI) scans looking for bone 
breaching and radiolucent lines around the device, as well as with dynamic radiographs to assess any translational movement of the device. In our series, $90 \%$ of patients did not exhibit any evidence of bone breaching or radiolucency around the device, with translational movement of less than $2 \mathrm{~mm}$ on dynamic radiographs at the 1-year followup in all patients. Furthermore, MRI scans confirmed restoration of cervical lordosis, with maintenance over the follow-up period (Fig. 2).

There was no persistent dysphagia, voice complaints, dural tear, tracheal perforations, or oesophageal perforations. One patient developed a deep methicillin resistant Staphylococcus aureus (MRSA) infectious infarction of the

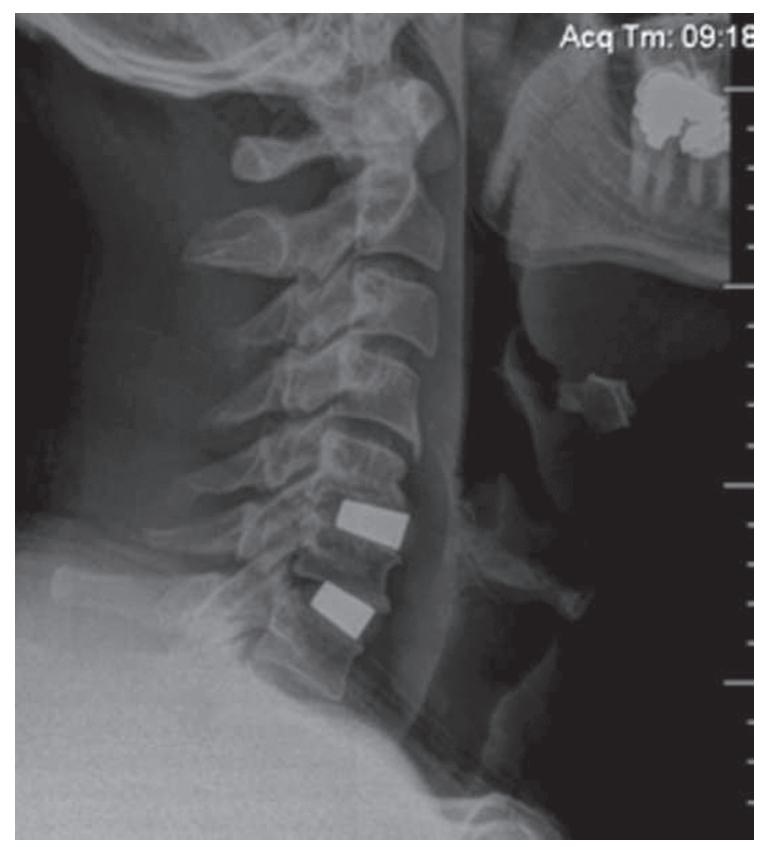

Fig. 1. Radiographic evidence of pseudoarthrosis.
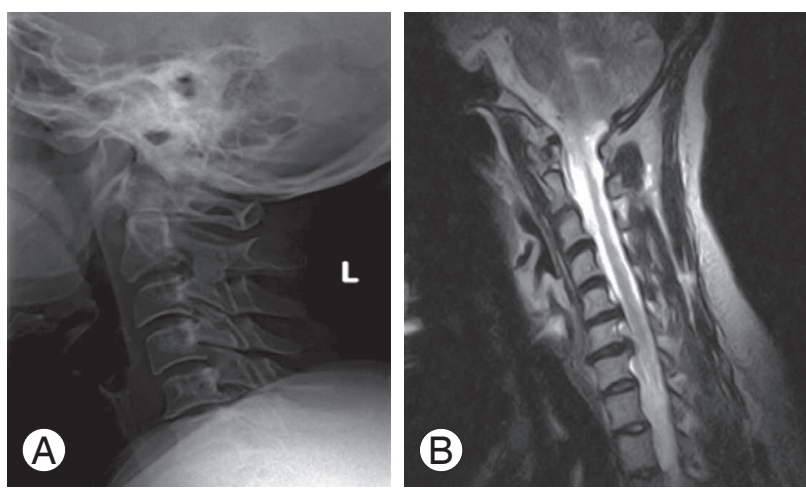

Fig. 2. Evidence of cervical lordosis restoration and its maintenance. (A) Preoperative lateral radiograph. (B) Preoperative sagittal magnetic resonance imaging (MRI). (C) Preoperative axial MRI. (D) Postoperative lateral radiograph. spinal cord, which was treated with antibiotics. Recovery was complete when reviewed at the 1-year follow-up [11].

Four patients $(3.6 \%)$ had to undergo further cervical discectomy and fusion surgery planned at the final followup. All these patients presented with symptomatic progressive cervical spondylosis, not related to the previously operated segments, and did not respond to conservative measures for at least 6 weeks.

\section{Discussion}

Trabecular metal cages (Fig. 3) provide an excellent balance between porosity and strength. They offer a high coefficient of friction to help prevent device migration and extrusion, as well a low modulus of elasticity that may improve load-sharing, which potentially minimizes stress shielding. With their average porosity of $80 \%$ and a

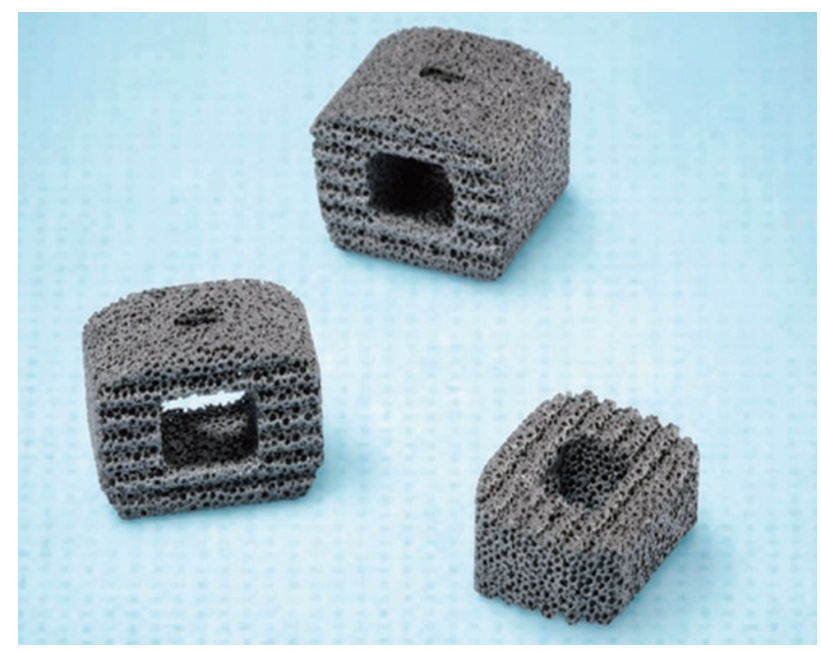

Fig. 3. Trabecular metal cages.
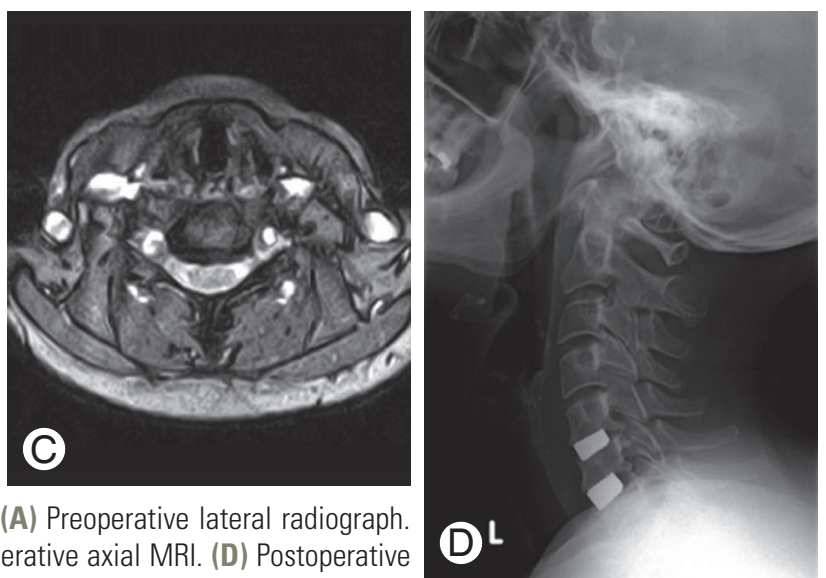
consistent open pore structure, they are also designed to resemble the structure and mechanical properties of cancellous bone, which provides an excellent environment for boney in-growth and vascularization.

Traditionally, autologous iliac bone graft has been used to fill the disc space during ACDF, with good fusion rates and clinical outcomes. However, autologous bone graft comes with its own set of donor site morbidities including pain, numbness, and infection $[2,12,13]$. As a result, the use of stand-alone cages has become popular, with many reports describing its effectiveness with good stability, restoration of disc height, and facilitation of bone fusion.

Nonunion and subsidence have been reported as complications of ACDF using stand-alone cages [14,15]. Overdistraction, damage of endplate, and osteoporosis are risk factors for subsidence and nonunion [16]. Adequate preparation of both endplates so as not to damage the bony cartilage is an important factor in decreasing subsidence after ACDF with stand-alone cage. In our series, the subsidence rate was $8 \%$ of levels fused, and the radiological finding of subsidence did not correlate with a worse clinical outcome, and we found no cage migration or extrusion at the 1- and 3-year follow-up, as an indirect measure of union and fusion.

The main presenting symptom in our series was radiating pain (83\%) to either arm, similar to other studies $[17,18]$. Seventeen percent of patients complained of axial neck pain, recurrent headaches, dizziness, and sclerodermal radiation to the shoulder. At the 1- and 3-year follow-up, the improvement of axial neck pain was $80 \%$ and $75 \%$ respectively, and of radicular arm pain was $95 \%$ and $85 \%$ of patients respectively. Patients with a preoperative upper extremity weakness and numbness tended to more frequently develop postoperative motor and sensory deficits at the final follow-up period following an ACDF procedure. It is likely that these deficits either represent a recurrence of preoperative deficits or more likely, an overlap of dermatomes and myotomes from an adjacent or remote level supporting the notion of progression of cervical spine degenerative disease, acting as a major contributor to new postoperative sensory and motor deficits [19].

In our series, there was a significant improvement in patient VAS scores at 1 year, with this improvement maintained at 3 years postoperatively. Fifty one patients underwent 2-level ACDF, and 39 patients underwent 1-level ACDF, usually at the C5/C6 (65\%), consistent with the results of other studies $[18,20]$.
Recently, Mehra et al. [21] found that approaching spinal levels above $\mathrm{C} 4$ and exposing more than 3 spinal levels were two factors significantly related to voice and swallowing problems. As all our operative levels were below $\mathrm{C} 4$ and we did not expose 3 levels in our patients, we did not have any incidence of persistent dysphagia or voice complaints.

One patient developed a deep MRSA infection after C6/C7 ACDF leading to cord ischemia, with radiological changes and rapidly evolving neurological deterioration. Rapid evaluation with MRI scanning and initiation of antibiotic produced dramatic response with our patient and total recovery at the 1-year follow-up [11].

With ACDF procedures, there are risks of adjacent segments degenerating [22]. This has been observed in approximately $3 \%$ of patients in a retrospective long-term follow-up study, with a predicted prevalence at 10 years of approximately 25\% [23]. Similar findings were observed in our series at the final follow-up. Recently, a meta-analysis indicated that the mid- to long-term rates of adjacent segment disease and neurological improvements were not significantly different when cervical disc arthroplasty was compared with anterior discectomy and fusion [24]. More importantly, there are no natural history studies yet available to demonstrate a difference between the likelihood of adjacent segment disease developing when ACDF, cervical disc arthroplasty, and non-operated yet symptomatic cervical spondylosis are compared.

The reoperation rate of our series was $3.6 \%$, which is comparable to the rate of the 5 -year results published by the Food and Drug Administration ProDisc-C study [25].

\section{Conclusions}

To our knowledge, this is the first publication reporting specifically on the mid-term outcomes of ACDF with stand-alone trabecular metal cages as treatment for cervical radiculopathy. Excellent and/or good outcomes were evident in ACDF with acceptable low reoperation rates, and reduced operative times and complications. This appears to be a safe and effective option in treating single and 2-level cervical disc radiculopathy and neck pain.

\section{Conflict of Interest}

No potential conflict of interest relevant to this article was reported. 


\section{References}

1. Rhee JM, Yoon T, Riew KD. Cervical radiculopathy. J Am Acad Orthop Surg 2007;15:486-94.

2. Smith GW, Robinson RA. The treatment of certain cervical-spine disorders by anterior removal of the intervertebral disc and interbody fusion. J Bone Joint Surg Am 1958;40:607-24.

3. Cloward RB. The anterior approach for removal of ruptured cervical disks. J Neurosurg 1958;15:602-17.

4. Ba Z, Zhao W, Wu D, Shen B, Yu B, Wang Z. Box cages packed with local decompression bone were efficient in anterior cervical discectomy and fusion: five- to 10-year follow-up. Spine (Phila Pa 1976) 2012;37:E1260-3.

5. Kaiser MG, Haid RW Jr, Subach BR, Barnes B, Rodts GE Jr. Anterior cervical plating enhances arthrodesis after discectomy and fusion with cortical allograft. Neurosurgery 2002;50:229-36.

6. Kulkarni AG, Hee HT, Wong HK. Solis cage (PEEK) for anterior cervical fusion: preliminary radiological results with emphasis on fusion and subsidence. Spine J 2007;7:205-9.

7. Vavruch L, Hedlund R, Javid D, Leszniewski W, Shalabi A. A prospective randomized comparison between the cloward procedure and a carbon fiber cage in the cervical spine: a clinical and radiologic study. Spine (Phila Pa 1976) 2002;27:1694-701.

8. Ha SK, Park JY, Kim SH, Lim DJ, Kim SD, Lee SK. Radiological assessment of subsidence in stand-alone cervical polyetheretherketone (PEEK) cage. J Korean Neurosurg Soc 2008;44:370-4.

9. Lee $\mathrm{CH}$, Hyun SJ, Kim KJ, et al. Comparative analysis of 3 different construct systems for single-level anterior cervical discectomy and fusion: stand-alone cage, iliac graft plus plate augmentation, and cage plus plating. J Spinal Disord Tech 2013;26:112-8.

10. Ji GY, Oh CH, Shin DA, et al. Stand-alone cervical cages versus anterior cervical plates in 2-level cervical anterior interbody fusion patients: analysis of adjacent segment degeneration. J Spinal Disord Tech 2015;28:E433-8.

11. Shawky A, El-Abed K. Spinal cord MRSA infectious infarction after anterior cervical decompression. J Spine 2013;S2:001.

12. Odom GL, Finny W, Woodhall B. Cervical disk lesions. JAMA 1958;166:23-8.
13. Banwart JC, Asher MA, Hassanein RS. Iliac crest bone graft harvest donor site morbidity: a statistical evaluation. Spine (Phila Pa 1976) 1995;20:1055-60.

14. Barsa P, Suchomel P. Factors affecting sagittal malalignment due to cage subsidence in standalone cage assisted anterior cervical fusion. Eur Spine J 2007;16:1395-400.

15. Yang JJ, Yu CH, Chang BS, Yeom JS, Lee JH, Lee CK. Subsidence and nonunion after anterior cervical interbody fusion using a stand-alone polyetheretherketone (PEEK) cage. Clin Orthop Surg 2011;3:16-23.

16. Park JI, Cho DC, Kim KT, Sung JK. Anterior cervical discectomy and fusion using a stand-alone polyetheretherketone cage packed with local autobone :assessment of bone fusion and subsidence. J Korean Neurosurg Soc 2013;54:189-93.

17. Clements DH, O'Leary PF. Anterior cervical discectomy and fusion. Spine (Phila Pa 1976) 1990;15:10235.

18. Acharya S, Kumar S, Srivastava A, Tandon R. Early results of one-level cervical discectomy and fusion with stand-alone cervical cage and bone marrow soaked tricalcium phosphate. Acta Orthop Belg 2011;77:218-23.

19. Lehmann CL, Buchowski JM, Stoker GE, Riew KD. Neurologic recovery after anterior cervical discectomy and fusion. Global Spine J 2014;4:41-6.

20. Hacker RJ, Cauthen JC, Gilbert TJ, Griffith SL. A prospective randomized multicenter clinical evaluation of an anterior cervical fusion cage. Spine (Phila Pa 1976) 2000;25:2646-54.

21. Mehra S, Heineman TE, Cammisa FP Jr, Girardi FP, Sama AA, Kutler DI. Factors predictive of voice and swallowing outcomes after anterior approaches to the cervical spine. Otolaryngol Head Neck Surg 2014;150:259-65.

22. Lundine KM, Davis G, Rogers M, Staples M, Quan G. Prevalence of adjacent segment disc degeneration in patients undergoing anterior cervical discectomy and fusion based on pre-operative MRI findings. J Clin Neurosci 2014;21:82-5.

23. Hilibrand AS, Carlson GD, Palumbo MA, Jones PK, Bohlman HH. Radiculopathy and myelopathy at segments adjacent to the site of a previous anterior cervical arthrodesis. J Bone Joint Surg Am 1999;81:51928.

24. Ren C, Song Y, Xue Y, Yang X. Mid- to long-term 
outcomes after cervical disc arthroplasty compared with anterior discectomy and fusion: a systematic review and meta-analysis of randomized controlled trials. Eur Spine J 2014;23:1115-23.

25. Zigler JE, Delamarter R, Murrey D, Spivak J, Janssen
M. ProDisc-C and anterior cervical discectomy and fusion as surgical treatment for single-level cervical symptomatic degenerative disc disease: five-year results of a Food and Drug Administration study. Spine (Phila Pa 1976) 2013;38:203-9. 